achieved an AUC of 0.71 was used as our final model. For validation on the testing dataset, the model yielded an accuracy of $90 \%$ and AUC of 0.93 (95\% CI: 0.76 to 1.00 ) while the percentage of patches positively classified, and outperforms average of the probabilities of the corresponding patches (accuracy 70\%; AUC 0.79, 95\% CI: 0.50 to 1.00) using the same optimal threshold of 0.33 . The heatmaps show that almost all of patches are highly identified to show the regions of immunoscore ((figure 1) A. Immunoscore of 3-4 [positive]. B. Immunoscore of $0-2$ [negative]).

Conclusions The automated deep-learning model achieved good performance and could potentially assist clinicians in the identification of HCC patients who are more likely to respond to immunotherapy, or at least, providing second opinions on therapeutic decision-making.

\section{IDDF2020-ABS-0088 COMPARING NON-INVASIVE TESTS FOR PREDICTION OF FIBROSIS IN NON- ALCOHOLIC FATTY LIVER DISEASE}

Wilson Siu*, Yusuke Onishi, Ashis Mukhopadhya. Department of Digestive Disorders, Aberdeen Royal Infirmary, UK

\subsection{6/gutjnl-2020-IDDF.151}

Background Non-alcoholic fatty liver disease (NAFLD) is becoming the most common type of chronic liver disease with an estimated worldwide prevalence of $25 \%$. It is a spectrum of disease that ranges from simple steatosis to advanced fibrosis. Non-invasive tests play an important role in identifying patients with fibrosis that require further investigation and follow up. The aim of the study is to evaluate the diagnostic accuracy of different non-invasive scoring tests.

Methods Patients with NAFLD who underwent liver stiffness measurement (LSM) by FibroScan at Aberdeen Royal Infirmary between 2013 and 2016 were retrospectively included in our study. Patients' demographic, clinical and laboratory data were collected closest from the date of the FibroScan. NAFLD fibrosis score (NFS), APRI, FIB-4 and BARD scores were calculated. For this study, clinically significant fibrosis (CSF) is defined as LSM $>7 \mathrm{kPa}$ and advanced fibrosis is defined as LSM $>12 \mathrm{kPa}$. The diagnostic accuracy of the four fibrosis scores was examined by calculating the area under the receiver operating characteristic curve (AUROC). The sensitivity, specificity, positive predictive value and negative value were calculated using optimal cut-offs calculated by Youden index.

Results Of the 863 patients included in this study, 498 $(57.7 \%)$ were male and the mean age was 54.4 years $(\mathrm{SD}=14.7)$. The mean $\mathrm{BMI}$ was $32.6 \quad(\mathrm{SD}=6.4) .48 \%$ of patients had CSF with LSM $>7 \mathrm{kPa}$ and $28 \%$ had advanced fibrosis with LSM $>12 \mathrm{kPa}$. For CSF, AUROC curve values were: NFS 0.77 (95\% CI, 0.73-0.80), FIB-4 0.74 (95\% CI, $0.71-0.78)$, APRI 0.74 (95\% CI, 0.70-0.78) and BARDS 0.65 (95\% CI, 0.65-0.74). For advanced fibrosis, the AUROC curve values were: NFS 0.83 (95\% CI, 0.80 - 0.87), FIB-4 0.79 (95\% CI, 0.75-0.83), APRI 0.75 (95\% CI, 0.71 to 0.79 ) and BARDS 0.75 (95\% CI, 0.71-0.79).

Conclusions All fibrosis scores were superior at detecting advanced fibrosis (LSM>12kPa) compared to CSF (LSM > $7 \mathrm{kPa})$. NFS showed a superior diagnostic accuracy of fibrosis compared to other scores.

\section{IDDF2020-ABS-0092 PREDICTION OF POST-HEPATECTOMY LIVER FAILURE IN PATIENTS WITH HEPATOCELLULAR CARCINOMA BASED ON GD-EOB-DTPA-ENHANCED MRI: A LF SCORING MODEL}

Yuyan Chen*. The First Affiliated Hospital of Sun Yat-sen University, China

\subsection{6/gutjnl-2020-IDDF.152}

Background The purpose of this study was to establish a preoperative clinical-radiomics prediction model of post-hepatectomy liver failure (PHLF) in patients with hepatocellular carcinoma(HCC) and to predict clinical outcome of HCC patients who suffered PHLF.

Methods The study included 555 HCC patients who underwent hepatectomy first time from January 2015 to December 2019 in The First Affiliated Hospital of Sun Yat-sen University and Sun Yat-sen University Cancer Center. Gd-EOB-DTPAEnhanced MRI was performed within 30 days before surgery. Patients in this study didn't have other tumors or serious organic diseases and were followed up after liver resections for 90 days. We obtained 60720 MR images from 555 patients (an ROI is shown as figure 1), including 390 cases as training data and 165 cases as independent testing data with the standard of PHLF as ISGLS. Through the multiple-model fusion algorithm, we extracted 1044 features per patient from his MR images, screening out feature sets of high contribution by RFE-SVM algorithm and transforming them to FF scores. Clinical indicators, radiologic features and FF scores were included in our LF scoring model through LDA(Linear discriminant Analysis) algorithms.

Results The AUC of LF scoring model reached 0.953 (95\%CI $0.953-0.963)$ and 0.945 (95\%CI $0.941-0.980)$ in the training

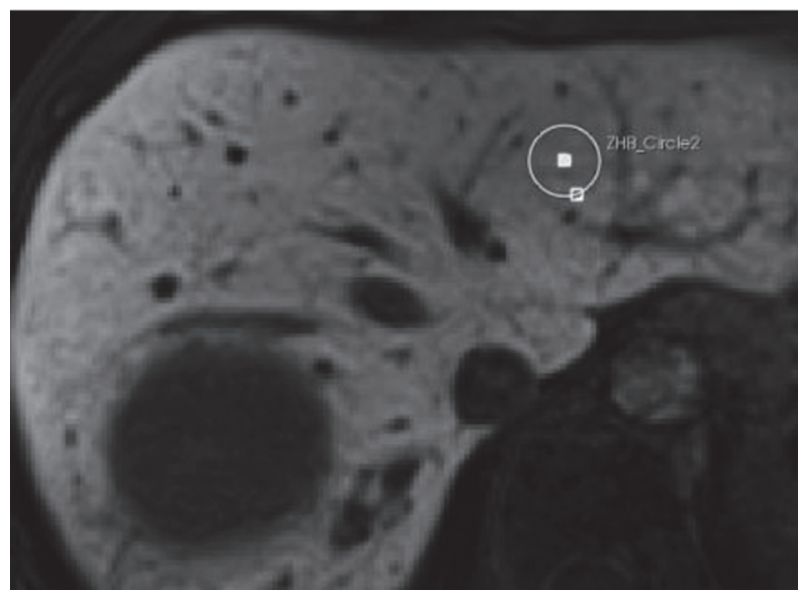

Abstract IDDF2020-ABS-0092 Figure 1 ROI of a certain patient

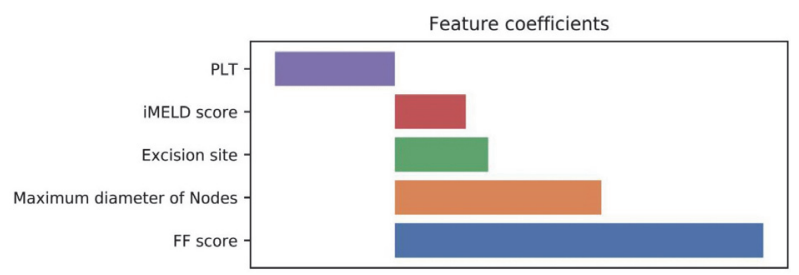

Abstract IDDF2020-ABS-0092 Figure 2 Feature coefficients in the clinical-radiomics model 


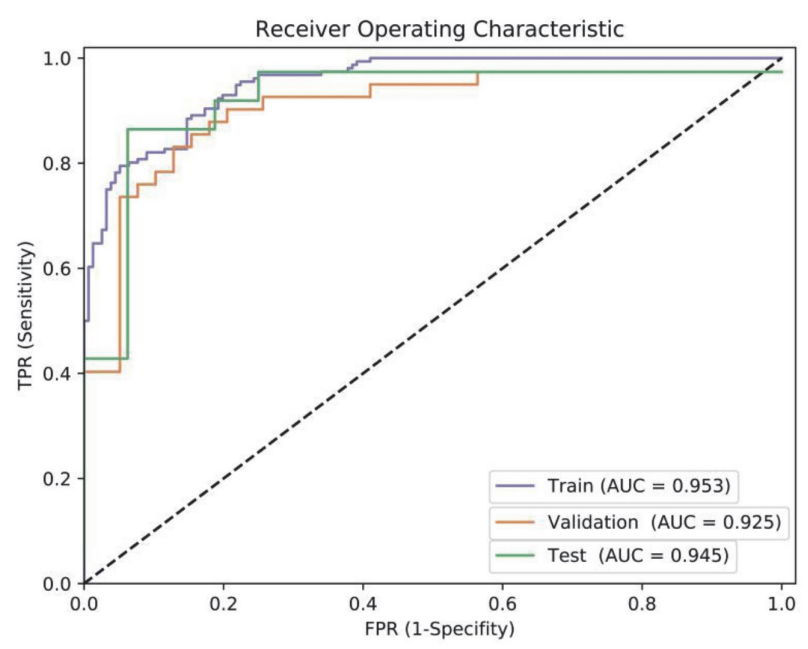

Abstract IDDF2020-ABS-0092 Figure 3 ROC curve of the clinicalradiomics model

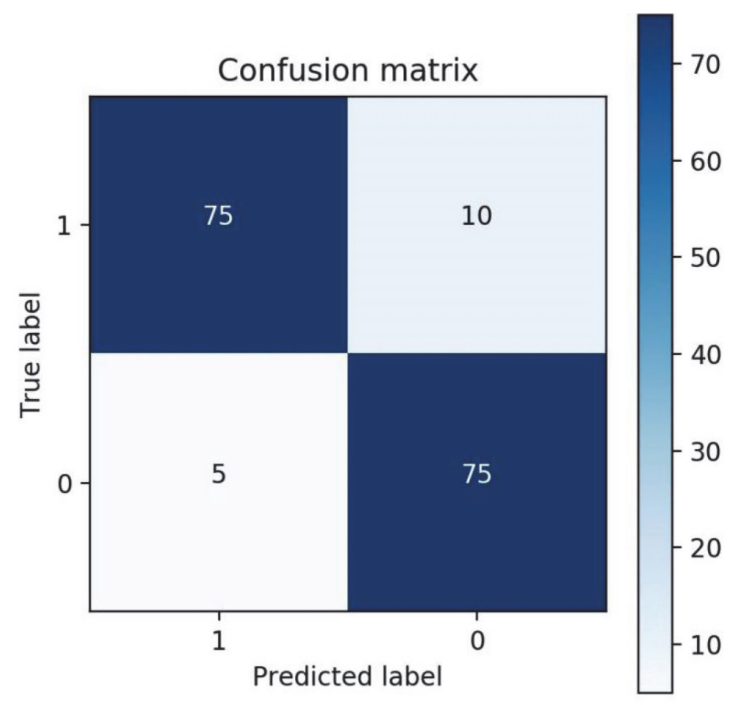

Abstract IDDF2020-ABS-0092 Figure 4 Confusion matrix of the clinical-radiomics model set and the testing set, respectively (Results are shown in figure 3 and figure 4). The model showed the maximum diameter of nodes, PLT, excision site,iMELD score and FF score were independent risk factors of PHLF in HCC patients $(\mathrm{OR}=1.258,0.986,4.670,1.237,320.382 ; \mathrm{P}<0.05$, and feature coefficients shown as figure 2). Nomogram by Logistic Regression with predictors is shown in figure 5. Moreover, the AUC of LF scoring model was significantly higher than clinical-imaging model and single radiomics model. Meanwhile, the accuracy of the model in predicting overall survival and progression-free survival of HCC patients with PHLF were 0.771 and 0.762 , respectively.

Conclusions The study indicates that LF scoring model has a good predictive value for the occurrence of PHLF in HCC patients and plays a vital role in predicting the clinical outcome of HCC patients who suffered PHLF.

\section{IDDF2020-ABS-0103 EFFICACY AND SAFETY OF PROGRAMMED CELL DEATH PROTEIN 1 INHIBITOR AND THE ASSOCIATED PROGNOSTIC FACTORS IN PATIENTS WITH HEPATITIS B VIRUS- RELATED ADVANCED HEPATOCELLULAR CARCINOMA}

Lingeng Wu*, Wei Cui, Qing Gou, Zejian Zhou. Guangong Province People's Hospital, China

\subsection{6/gutjph-2020-IDDF.153}

Background Programmed cell death protein 1 (PD1) inhibitor is safe and effective for hepatocellular carcinoma (HCC) treatment; however, the correlation between previous hepatitis $\mathrm{B}$ virus (HBV) infection and the clinical outcomes of PD1 treatment remain unclear. This study evaluated the safety and efficacy of PD-1 inhibitor treatment for HBV-related advanced HCC and determined the associated prognostic factors.

Methods Fifty HBV-infected HCC patients treated with PD-1 inhibitor in a clinical trial were retrospectively investigated. Treatment responses as per the response evaluation criteria in solid tumors 1.1 (RECIST 1.1) and immune-modified RECIST criteria (imRECIST). Overall survival (OS) and time to progression (TTP) were evaluated, and any adverse events (AEs) were recorded.

Abstract IDDF2020-ABS-0092 Figure 5 Nomogram by logistic regression with predictors 